\title{
Les anticorps monoclonaux
}

\section{L'histoire d'une recherche fondamentale ou la curiosité comme source de richesse ${ }^{1}$}

> En 2019, les anticorps monoclonaux (Acm) vont représenter un marché mondial annuel de plus de cent milliards de dollars, soit près de 90 milliards d'euros. Outre leur utilisation en clinique, les anticorps monoclonaux sont utilisés également dans de nombreux tests diagnostiques et sont toujours des outils précieux pour la recherche fondamentale et appliquée. Quarantequatre ans après la publication de Georges Köhler et César Milstein [1], des dizaines de congrès et séminaires de toute nature sur les anticorps monoclonaux se tiennent annuellement à travers le monde. Mais 44 ans plus tard, les travaux scientifiques qui ont amené à cette publication sont peu à peu oubliés et, dans bien des esprits, les anticorps monoclonaux ne sont qu'un business d'un multi-milliard euros/dollars comme un autre, déterminé par les marchés financiers et les résultats des derniers essais cliniques... II est grand temps de rendre hommage à toute une génération de chercheurs fondamentalistes, à ces fous de science du $\mathrm{xx}^{\mathrm{e}}$ siècle, à ces chercheurs connus et souvent désormais méconnus, disséminés aux quatre coins du monde, qui ont exploré les frontières de l'inconnu d'alors et qui ont modelé et ciselé un savoir qui a débouché sur une technique d'obtention de molécules qui ont permis l'une des plus grandes révolutions thérapeutiques de ces vingtcinq dernières années. <

\section{Acte I : le début de la longue histoire des anticorps, de Paul Ehrlich à Karl Landsteiner}

En 1975, il n'y avait pas encore de start-up et, ce qu'il est communément convenu d'appeler les big pharma, ne s'intéressaient pas aux prémices de ce qui allait devenir les biotechnologies. Mais en 1975, il y avait, à Cambridge au Royaume-Uni, l'un de ces fous de science que le $x^{e}$ siècle

${ }^{1}$ «La curiosidad como fuente de riqueza» a été le sous-titre de la conférence « Los anticuerpos monoclonales » donnée par César Milstein à l'université de Buenos Aires (Argentine) le 15 décembre 1999.
Béré K. Diallo, Clémence Riffard, Kenz Le Gouge, Jean-Luc Teillaud

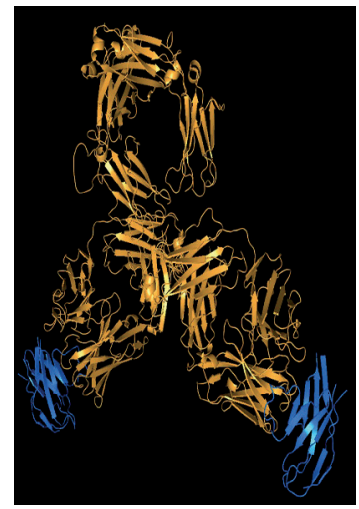

Sorbonne Université, Inserm U1135, CNRS ERL8255, Centre d'Immunologie et des Maladies Infectieuses (CIMI), 91 boulevard de l'hôpital, 75013 Paris, France. jean-luc.teillaud@inserm.fr

a connu, argentin émigré, César Milstein. César avait obtenu en 1957 un doctorat de chimie portant sur la cinétique enzymatique de l'aldéhyde déshydrogénase à l'université de Buenos Aires (célèbre sous son acronyme, la UBA), puis avait effectué un premier séjour au Medical Research Council (MRC) britannique à Cambridge. Rentré en Argentine en 1961, il avait émigré de nouveau en 1963 après la prise de pouvoir par les militaires en 1962 et rejoint Frederick Sanger, deux fois récipiendaire du prix Nobel de Chimie (en 1958 et en 1980), au laboratoire de biologie moléculaire du MRC qui venait d'être créé et était dirigé alors par un autre prix Nobel de Chimie (1962), Max Perutz. Ce retour à Cambridge s'accompagna d'un changement thématique radical sous les conseils de Sanger, un passage du monde de l'enzymologie au monde de l'immunologie moléculaire, à un moment où la recherche pour comprendre les bases moléculaires de la diversité et de la spécificité des anticorps s'accélérait. À la fin du xIx ${ }^{e}$ siècle et au début du Xx $x^{e}$ siècle, Paul Ehrlich ${ }^{2}$ avait jeté à Berlin les bases des études moléculaires des interactions antigène-anticorps (Figure 1) en développant une immunochimie quantitative qui avait permis de titrer [2] et de rendre ainsi plus efficaces les sérums antidiphtériques d'Emil von Behring et Shibasaburo Kitasato destinés à être vendus par la firme Hoechst, à la suite d'un accord passé par von Behring avec celle-ci, accord excluant Ehrlich, ce qui conduisit à une brouille tenace entre les deux allemands et démontre que même des « fous de science » savent avoir un regard attentif sur la valorisation industrielle !

${ }^{2}$ Prix Nobel de Physiologie ou Médecine qu'il partagea avec Mechnikov (dont le nom en alphabet latin est Elie Metchnikoff) en 1908. 


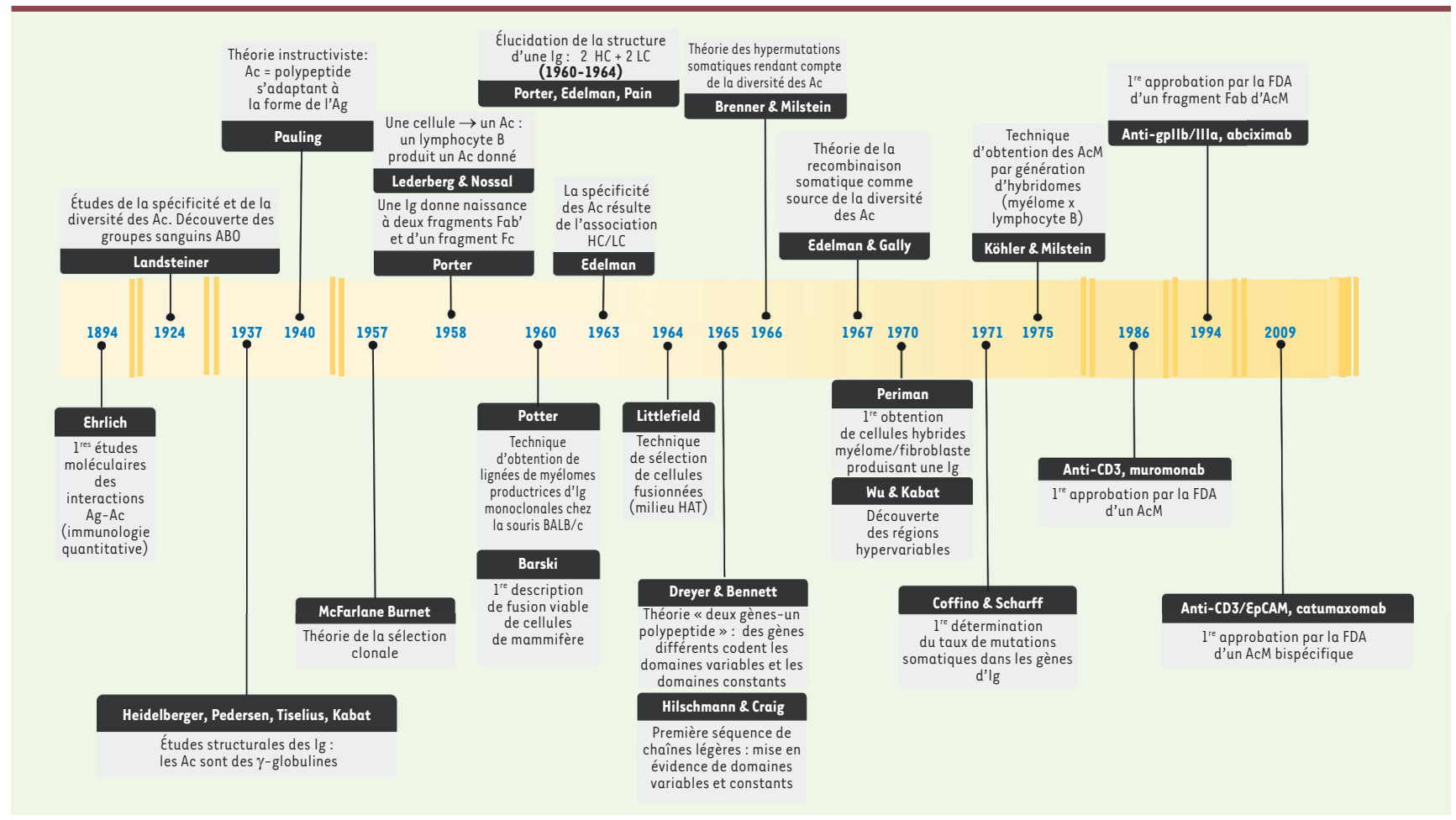

Figure 1. Quelques dates clés de l'histoire de la recherche sur les anticorps conduisant à l'élaboration de la technique des anticorps monoclonaux et à différents formats thérapeutiques. Ac : anticorps ; AcM : anticorps monoclonal ; Ag : antigène ; Fab : fragment antigen-binding ; Fc : fragment cristallisable ; FDA : food and drug administration; HAT : hypoxathine-aminoptérine-thymidine; HC : heavy chain ; HL : light chain ; Ig : immunoglobuline.

Les travaux de Karl Landsteiner ${ }^{3}$, menés à New York au cours des années 1920, avaient ensuite montré la capacité extraordinaire des anticorps à être dirigés contre un nombre quasi illimité de molécules chimiques, synthétiques ou naturelles [3]. Landsteiner démontra notamment qu'il était possible d'obtenir des anticorps capables de distinguer de très légères modifications chimiques d'une même molécule, dues au couplage de groupements chimiques à celle-ci, inventant au passage le concept d'haptènes ${ }^{4}$ [4]. Ces études fondamentales conduisirent à la découverte des groupes sanguins, grâce à des tests d'agglutination entre sérums de donneurs et globules rouges de donneurs, ce qui lui valut le prix Nobel (Figure 1). Les travaux de Landsteiner donnèrent également naissance, dans les décennies suivantes, à toute une série d'immuno-essais fondés sur l'utilisation des anticorps dont l'un des tout-premiers, l'immuno-électrophorèse, fut développé par Pierre Grabar et Curtis Williams [5].

\section{Acte II : élucider la structure des anticorps et comprendre les bases moléculaires de leur diversité de reconnaissance}

Ces premiers développements d'immunochimie moléculaire donnèrent naissance aux travaux d'une nouvelle génération de scientifiques qui s'attelèrent à l'élucidation de la structure des anticorps pour comprendre

\footnotetext{
${ }^{3}$ Prix Nobel de Physiologie ou Médecine en 1930.

${ }^{4}$ Un haptène est une petite molécule incapable d'induire la production d'anticorps contre elle-même sauf si celle-ci est couplée chimiquement à une grosse molécule dite «porteuse » (carrier). L'haptène devient alors immunogénique et induit l'apparition d'anticorps spécifiquement dirigés contre lui.
}

l'origine de leur diversité et de leur spécificité (Figure 2). Il fallut d'abord comprendre la nature chimique des anticorps, et ceci fut réalisé entre 1937 et 1939 grâce à Michael Heidelberger, Kai Pedersen, Arne Tiselius et Elvin Kabat (Figure 1) $[6,7]$. Un signe d'égalité fut alors mis entre la notion d'immunoglobulines (Ig) et celle d'anticorps, les seconds n'étant que l'appellation fonctionnelle des premières lorsqu'une spécificité de reconnaissance d'un antigène bien défini leur est attribuée.

Lorsque César Milstein débuta ses travaux sur les anticorps en 1963, de nombreuses autres connaissances sur la structure des anticorps et les cellules qui les produisaient, normales ou pathologiques, étaient venues enrichir le monde naissant de l'immunologie moderne. D'une part, l'idée que les antigènes « instruisaient » la forme des anticorps, pour que ceux-ci puissent les fixer, avait été largement battue en brèche au profit de la théorie clonale élaborée par Frank McFarlane Burnet en 1957 [8], qui stipulait qu'un lymphocyte $B$ exprimait un récepteur spécifique et donnait naissance après stimulation à une population de cellules-filles (un clone) produisant un anticorps de même spécificité (Figure l et Figure 2B). L'année suivante, Gustav Nossal et Joshua Lederberg (Figure 1) apportèrent la preuve expérimentale de cette théorie, en montrant qu'une cellule $B$ isolée provenant d'un rat immunisé avec 
deux immunogènes différents ne produisait des anticorps que contre I'un de ces antigènes et non contre les deux [9]. Dame Nature se portait ainsi au secours de la mise au point de la technique des anticorps monoclonaux! D'autre part, Linus Pauling avait proposé en 1940 un modèle où les Ig étaient constituées d'un polypeptide composé de trois parties, l'une rigide et centrale, encadrée par deux extrémités flexibles ayant toujours la même séquence mais capables d'adopter une conformation complémentaire à celle de l'antigène ciblé (Figure 1 et Figure 2A) [10]. Ce modèle avait servi de base au Iondonien Rodney Porter ${ }^{5}$ qui, à l'aide de remarquables expériences de digestion par la papaïne de $\gamma$-globulines ( $\lg G)$ de lapin anti-ovalbumine ou anti-albumine bovine ou humaine, avait montré qu'une Ig pouvait être digérée en trois fragments, l'un identique, d'une Ig à l'autre et dépourvu d'activité de reconnaissance, cristallisable (la fraction III, ultérieurement appelée fragment Fc), les deux autres capables de lier l'antigène (les fractions I et II, appelées plus tard Fab') (Figure 1) [11]. Une nouvelle étape fût franchie en 1959 lorsque Porter établit les masses moléculaires de ces différents fragments ainsi que leur composition en sucres, avec son collègue londonien Henry Perkins qui montra que la plus grande partie de la glycosylation était associée à la fraction III (le fragment Fc) [11]. Porter put aussi établir que ces fractions présentaient des compositions en acides aminés différentes, grâces à ses collègues Elaine McDermott et John Pace : les fractions I et II étaient quasiment identiques, mais très différentes de la fraction III [11]. En cette fin des années 1950, la structure précise des lg et les bases moléculaires à l'origine de leur diversité de reconnaissance restaient néanmoins encore largement inconnues. Le modèle avancé alors par Porter était celui d'une molécule constituée de trois composants associés les uns aux autres, dont deux très identiques et un troisième différent [12]. C'est en 1961 que Gerald Edelman et Miroslov Poulik montrèrent que les $\gamma$-globulines étaient composées de plusieurs polypeptides liés par des ponts disulfures, en utilisant des immunoglobulines polyclonales ainsi que des immunoglobulines de myélome et de macroglobulinémie de Waldenström ${ }^{6}$ [13]. En 1962, Porter proposa alors une représentation graphique d'une Ig de lapin telle qu'on la connaît aujourd'hui, avec deux chaînes A et deux chaînes B [14] et, en 1963, Robert Pain confirma qu'une $\gamma$-globuline était effectivement constituée de deux grandes chaînes (les chaînes lourdes, H pour «heavy »), et de deux petites chaînes (les chaînes légères, L pour « light ») (Figure 1) [15]. La position du site de liaison à l'antigène commença à être suspectée $[16,17]$. La même année, Edelman $^{7}$ proposa un modèle visionnaire de la structure des anticorps, fondé sur de remarquables expériences de biochimie montrant que le site de liaison à l'antigène résulte de l'association d'une chaîne légère avec une chaîne lourde (Figure 1) [18]. Ces expériences montrèrent, de plus, que chaînes lourdes et légères de deux espèces animales différentes pouvaient se réassocier et permettre de retrouver la spécificité initiale des anticorps parentaux [19]. Des compositions en acides aminés détaillées furent également déterminées en 1962 par Edelman et son étudiant Joe Gally,

\footnotetext{
${ }^{5}$ Prix Nobel de Physiologie ou Médecine en 1972 avec Gerald Edelman.

${ }^{6}$ La macroglobulinémie de Waldenström (MW) est une prolifération lymphocytaire B indolente caractérisée par l'accumulation des cellules monoclonales dans la moelle osseuse et dans les tissus lymphoïdes périphériques, et associée à la production d'immunoglobulines $M(\operatorname{IgM})$ monoclonales sériques.

${ }^{7}$ Prix Nobel de Physiologie ou Médecine en 1972 avec Rodney Porter.
}

en utilisant des protéines de myélome et de Bence-Jones ${ }^{8}$, ce qui leur permit, de plus, de démontrer que ces dernières étaient des chaînes légères d'Ig de myélome [20].

Parallèlement, l'établissement d'une lignée de myélome murin transplantable chez la souris [21,22] et son étude in vitro montrant que ces cellules produisaient l'Ig du myélome [23] démultiplièrent les capacités d'analyse structurale des $\mathrm{lg}$. Les protéines de myélome devinrent alors des objets essentiels pour décrypter la structure des immunoglobulines grâce à leur purification en grandes quantités à partir du sérum de patients ou de souris. Issus de la transformation maligne de clones de lymphocytes $B$ en voie de différenciation terminale, les plasmablastes et les plasmocytes, les cellules de myélome produisent une Ig homogène, monoclonale, trouvée en grandes quantités dans le sérum des patients. Ces lg n'ont pas, dans leur très grande majorité, de spécificité antigénique connue. En 1960, Michael Potter, en s'appuyant sur les travaux de Ruth Merwin et Glenn Algire [24], établit une technique d'obtention de lignées de myélomes productrices d'Ig monoclonales chez la souris BALB/c (Figure 1) [25], technique qu'il simplifie en 1962 [26], tandis qu'il s'essaie à la mise en culture de ces tumeurs entre deux passages chez la souris [27]. En 1970, il devint possible de cultiver des cellules de myélome à long terme, grâce à Reuven Laskov et Matthew Scharff [28] et à Kengo Horibata et Alan Harris [29].

\section{Acte III : les années s'écoulent et il faut toujours élucider la structure des anticorps et comprendre les bases moléculaires de leur diversité de reconnaissance!}

Ces Ig monoclonales, qu'elles soient d'origine murine ou humaine, et les anticorps purifiés à partir d'animaux immunisés, vont être les outils utilisés pour déterminer la séquence en acides aminés des chaînes lourdes et légères et leur structure : en 1965, Claude Bennett et ses collègues du California Institute of Technology (Pasadena), en collaboration avec Potter, montrent que les chaînes légères de myélomes murins partagent une séquence « commune », mais que chacune d'entre elles présente également une séquence qui lui est spécifique [30]. Parallèlement, la première séquence d'une chaîne légère est rapportée par Norbert Hilschmann et Lyman Craig [31]. L'existence d'un domaine variable fusionné à un domaine constant pour qu'une chaîne légère soit élaborée venait d’être découverte ! Cette variabilité

\footnotetext{
${ }^{8}$ Les protéines de Bence Jones sont constituées de chaînes légères libres monoclonales d'immunoglobulines, d'isotype Kappa ou Lambda. Elles sont éliminées dans les urines en cas de gammapathie monoclonale. Leur présence contribue ainsi au diagnostic de certaines maladies (principalement le myélome).
} 


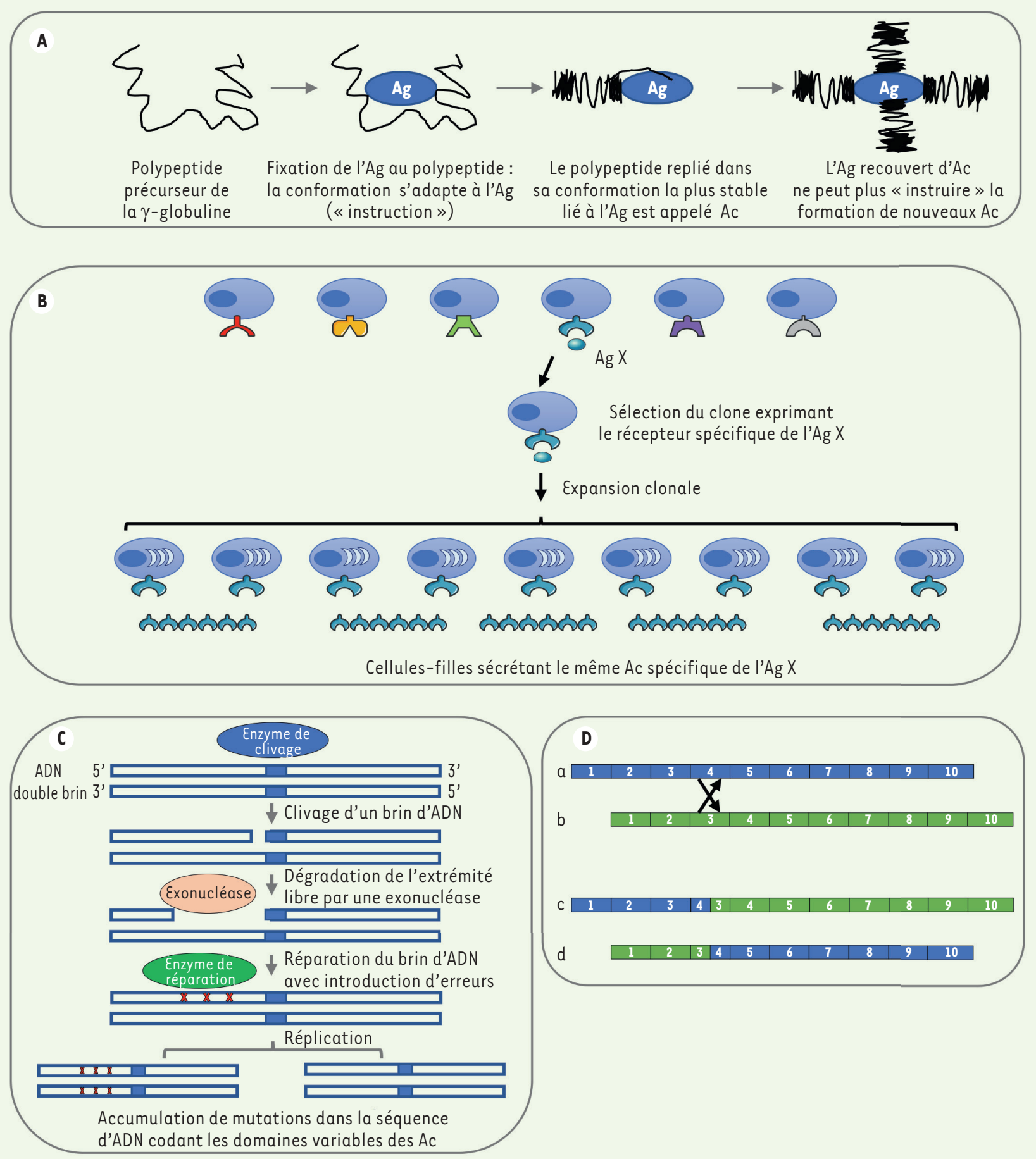

de composition en acides aminés, également observée dès 1963 par Marian Koshland et Frieda Englberger [32], puis détaillée par Jonathan Singer et Russell Doolittle en 1966 [33], amena Edelman et Gally à proposer l'existence d'un mécanisme de recombinaison somatique de gènes dupliqués permettant d'acquérir différentes spécificités (Figure 1 et Figure 2D) [34]. Le concept de recombinaison somatique des gènes codant les Ig était ainsi introduit dans les réflexions des immunochimistes pour expliquer l'origine de la diversité des anti-

corps. Un an auparavant, Sydney Brenner et Milstein avaient publié un article visionnaire dans lequel ils avaient émis l'hypothèse de l'existence d'un mécanisme d'hypermutation somatique restreint à un nombre limité de gènes, lié à la présence d'une enzyme coupant l'ADN en des sites précis, suivi d'erreurs lors du processus de réparation pour expliquer l'origine de la variabilité des anticorps (Figure 2C) [35]. 
4 Figure 2. Théories sur l'origine de la diversité de reconnaissance des anticorps. A. Théorie « instructive 》 de Linus Pauling en 1940 [10]. Dans cette théorie, l'antigène $(\mathrm{Ag})$ est délivré au site de synthèse du polypeptide précurseur de la $\gamma$-globuline, dont les extrémités ont une configuration instable et non repliée. L’Ag «attire » des séquences complémentaires de la $\gamma$-globuline grâce à des groupements chargés qu'il possède à sa surface, façonnant la conformation de la $\gamma$-globuline la plus complémentaire. Cette conformation s'adapte à celle de l'Ag pour rendre l'interaction la plus stable possible, notamment grâce à la flexibilité des extrémités de la $\gamma$-globuline. Pour Pauling, la $\gamma$-globuline est alors devenue un anticorps (Ac). Dans son modèle, il envisage deux sites de liaison à l'Ag par molécule de $\gamma$-globuline. B. Théorie de la sélection clonale de Burnet en 1957 [8]. Cette théorie, confirmée expérimentalement par Nossal et Lederberg l'année suivante (un lymphocyte $B$ = un anticorps), avançait l'hypothèse que chaque lymphocyte B exprime un récepteur spécifique, s'active après fixation de l'Ag correspondant, prolifère, conduisant à une expansion clonale de ce lymphocyte, donnant naissance à des cellules filles (clone) produisant le même anticorps contre cet Ag X. C. Théorie des hypermutations somatiques de Brenner et Milstein en 1966 [35]. Dans cette théorie visionnaire, une enzyme clive un brin de l'ADN du gène codant une chaîne légère, exposant une extrémité 3 '. Une exonucléase va ensuite dégrader une partie du brin d'ADN, exposant une partie de la séquence du brin complémentaire. Une ADN polymérase répare le brin d'ADN mais en introduisant des erreurs (délétions et/ou additions de nucléotides et erreurs d'appariements de base) lors de cette réparation, conduisant à l'apparition de mutations somatiques dans la chaîne légère. Le même processus surviendrait pour la chaîne lourde, expliquant ainsi l'origine de la diversité du répertoire des Ac. D. Théorie de la recombinaison somatique de gènes multiples dupliqués d'Edelman et Gally en 1966 [34]. Cette théorie fait l'hypothèse qu'il existe de nombreux gènes qui ont été dupliqués en tandem au cours de l'évolution et qui ont accumulé des mutations ponctuelles, ce qui va favoriser des entrecroisements («crossing-over ») somatiques entre eux au cours de la maturation du lymphocyte B, permettant d'acquérir une grande diversité de séquences d'acides aminés. Les recombinaisons somatiques ainsi que les erreurs d'appariements de bases sont facilitées par l'homologie des gènes et leur organisation en tandem. Edelman et Gally calculent que ces recombinaisons pourraient permettre de produire jusqu'à $10^{12}$ anticorps de spécificités différentes à partir de deux gènes (différents sur vingt nucléotides au minimum) codant les chaines lourdes et deux gènes (différents sur vingt nucléotides au minimum) codant les chaines légères. Dans le schéma, ( $a$ et $b$ ) montrent l'arrangement de 10 gènes avant recombinaison; ( $c$ et $\mathrm{d}$ ) montrent l'arrangement de ces gènes après recombinaison (adapté de la Figure 2 de [34]).

Les années suivantes virent un approfondissement des connaissances sur la variabilité trouvée dans les chaînes lourdes et légères [36], ainsi que sur les acides aminés directement impliqués dans les interactions avec les haptènes [37]. Des sous-groupes de variabilité des domaines variables des chaînes $L$ et $H$ commencèrent à être définis, ainsi que leurs tailles [38, 39]. L'idée qu'il existait plusieurs gènes codant les domaines variables et non pas un allélisme important touchant un seul gène, ainsi que celle de l'existence de deux types de gènes codant domaines variables et domaines constants firent ainsi leur chemin et furent brillamment théorisées par William Dreyer et Bennett sous la dénomination « deux gènes-un polypeptide » [40]. Il devint également évident qu'il existait des séquences encore plus variables... les régions hypervariables (HV) responsables de la complémentarité de l'anticorps avec son antigène, grâce aux travaux de Taï Wu et Kabat (Figure 1) [41]. Différentes écoles s'affrontaient donc, entre multiplicité de gènes et mutations somatiques. L'histoire nous apprendra que cela n'était pas incompatible... II devint également évident qu'il existait des régions constantes, dont la séquence ne différait entre groupes d'individus d'une même espèce, que par quelques acides aminés, différences déjà détectées à l'aide d'anticorps dits «anti-allotypes » par Jacques Oudin en 1960, ce qui lui avait permis de classer les immunoglobulines humaines selon ces allotypes [42] et d'ouvrir le champ de la génétique des immunoglobulines [43]. Cela l'amena à définir l'idiotypie, par une sérologie montrant que les domaines variables pouvaient être, eux-mêmes, immunogéniques et induire ainsi la notion d'anticorps anti-idiotypiques [44].

À la fin des années 1960, il était donc établi que les Ig étaient constituées de deux chaînes lourdes et de deux chaînes légères, faites de régions variables et de régions constantes (nommées en 1973 par Edelman domaines variables et domaines constants), que ces régions variables contenaient le site de liaison à l'antigène, et que la structure tertiaire des anticorps était fondée sur leur organisation en modules globulaires répétés. L'établissement par Edelman et ses collègues de la séquence complète d'une immunoglobuline en 1969 établit définitivement les caractéristiques structurales fondamentales des anticorps [45]. Il était également alors fermement établi que les immunoglobulines pouvaient être classées en fonction de leur isotypie (en classes et sous-classes), de leur allotypie et de leur idiotypie.

\section{Acte IV : quand les immunologistes rencontrent des cancérologues, des généticiens, des virologistes et des biologistes moléculaires !}

Les progrès réalisés dans l'élucidation de la structure des anticorps et de leurs sites de liaisons aux antigènes ouvrirent la porte à des études portant sur les mécanismes de synthèse et d'assemblage des chaînes $\mathrm{H}$ et L. Celles-ci visaient à comprendre en quoi cet assemblage contribuait à la diversité et à la spécificité des anticorps. Milstein porta d'abord ses efforts sur la formation et la localisation des ponts disulfures à l'origine de liaisons covalentes qui permettent la stabilisation des différents domaines variables et constants ainsi que l'association des chaînes $\mathrm{H}$ entre elles et des chaînes $H$ avec les chaînes $L$ [46]. Des progrès considérables sur la localisation et le nombre des ponts disulfures des immunoglobulines humaines et murines furent ainsi accomplis au cours de cette période. 
Mais la question des bases moléculaires de la diversité des anticorps et de sa relation avec leur structure restait toujours à résoudre. Un grand bond en avant fut effectué en 1971 lorsque Matthew Scharff et ses collègues montrèrent que l'on pouvait isoler, à l'aide d'une élégante technique de clonage de cellules de myélome en agar mou, des clones mutants avec une fréquence élevée $\left(1,1 \times 10^{-3}\right.$ par cellule et par génération) à partir des cellules du myélome murin MPC 11 (merwin plasma cell tumor-11), adaptées à la culture in vitro pour étudier initialement les règles de synthèse, d'association et de sécrétion de $\gamma$-globulines (Figure 1) [28, 47, 48]. Ces clones présentaient des mutations somatiques conduisant à la perte de production de la chaîne lourde de MPC 11 [48]. L'existence de la survenue de mutations somatiques dans les cellules de myélome cultivées in vitro fut confirmée deux ans plus tard par Milstein qui montra que des clones du myélome MOPC 21 (produisant une $\operatorname{lgGl}$, avec une chaîne lourde de type $\kappa$ ) cultivé in vitro, différant par leur point isoélectrique, pouvaient être isolés [49]. Deux articles vont alors être publiés et devenir fondateurs de la mise au point de la technique des anticorps monoclonaux : en décembre 1970, Phillip Periman obtint des cellules hybrides en fusionnant des cellules d'un myélome, MOPC 315, avec des fibroblastes de souris; selon lui, ces cellules produisaient toujours l'Ig de MOPC 315, bien qu'il ait eu beaucoup de difficultés à la détecter, ce qui l'amena à supposer que les cellules hybrides produisaient une Ig «altérée » (Figure 1) [50]. En mai 1971, Scharff et ses collègues [51] montrèrent que des cellules hybrides issues de la fusion des fibroblastes murins avec les cellules du myélome MPC 11 n'exprimaient plus I'Ig produite par ce dernier. Ils en conclurent qu'ils avaient un exemple de ce que Boris Ephrussi - l'un des fondateurs de la génétique somatique - considérait comme une re-différenciation conduisant à la perte des multi-potentialités de cellules parentales et de leurs caractéristiques phénotypiques [52]. Deux années plus tard, ce résultat amena Milstein et l'un de ses post-docs, Richard Cotton, à utiliser à nouveau l'hybridation cellulaire pour fusionner cette fois les cellules de deux lignées de myélome, l'une de souris (PlBul, dérivée du plasmacytome Adj-PC5), productrice d'une IgG2a $\kappa$ (avec un excès de chaînes $\kappa$ libres), l'autre de rat (210.RCY3.Agl), résistante à la 8 -azaguanine, produisant seulement une chaîne légère $\kappa$. Grâce à cette approche, Cotton et Milstein montrèrent que les cellules hybrides continuaient à produire toutes les chaînes des myélomes parentaux et que l'expression d'une des chaînes $L$ n'empêchait pas l'expression de l'autre ; en d'autres termes, que le phénomène d'exclusion allélique n'était pas dominant [53].

Mais d'où venait cette technique d'hybridation cellulaire? Pour répondre à cette question, il nous faut remonter quelques années plus tôt, en 1960, et nous transporter à ... Villejuif où l'un de ces « fous de science », Georges Barski, juif polonais rescapé de la Shoah qui avait choisi en 1945 la France et l'Institut Pasteur pour s'y spécialiser en virologie, étudiait la transformation maligne des cellules normales dans le laboratoire de culture de tissus et de virologie qu'il avait fondé à l'Institut Gustave Roussy. Voulant tester l'hypothèse d'un transfert de matériel génétique d'une cellule tumorale agressive vers une cellule « à très faible pouvoir tumoral », rendant celle-ci « hautement cancéreuse », il entreprit, avec Serge Sorieul et Francine Cornefert, de co-cultiver ces deux types de cellules (NCTC 2472 et NZTC 2555, toutes deux dérivées d'un clone de fibroblastes de souris) et observa l'apparition, au bout de 3-4 mois, de cellules qu'il caractérisa grâce à l'étude de leur caryotype comme étant des cellules hybrides provenant de la fusion des deux cellules parentales (Figure 1) [54].

La lecture de la fin de la communication qu'il fît alors à l'Académie des sciences française montre l'intelligence visionnaire de Barski : il y souligne les deux difficultés majeures auxquelles va être confrontée la fabrication des cellules hybrides: la très grande rareté de la fusion spontanée lors de la co-culture et l'avantage sélectif que doit avoir la cellule hybride sur les cellules parentales. Les généticiens, qui se sont emparés de l'observation de Barski pour développer la génétique somatique des cellules de mammifères, au premier rang desquels Boris Ephrussi, à Gif-sur-Yvette, ont besoin de disposer d'une technique de sélection pour se débarrasser des cellules parentales non fusionnées. En 1964, John Littlefield, un généticien américain, apporte la solution (Figure 1) [55]: les cellules eucaryotes disposent de deux voies de synthèse des nucléotides, une voie de néosynthèse à partir de sucres et d'acides aminés et une voie alterne, à partir de nucléosides. Des enzymes comme l'hypoxanthine-guanine phosphoribosyl-transférase (HGPRT ou HPRT) ou la thymidine kinase (TK) assurent des étapes essentielles de la voie alterne, la catalyse de la réaction entre une base purique et un phosphoribosyl pyrophosphate conduisant à la formation d'un nucléotide purique monophosphate. Leur déficience conduit à I'utilisation de la voie de néo-synthèse qui peut être bloquée par des drogues telles que l'aminoptérine, la 8-azasérine ou le méthotrexate. Les cellules déficientes en HGPRT ou TK se multiplient dans un milieu de culture normal mais, en présence d'un milieu contenant de l'aminoptérine (milieu HAT [hypoxanthine, aminoptérine, thymidine]) qui bloque l'unique voie de synthèse des nucléotides qu'il leur reste, meurent (Figure 3). L'hypoxanthine exogène présente dans le milieu de sélection permet de satisfaire les besoins en purines. Le milieu contient également de la thymidine parce que l'aminoptérine est un anti-folate qui bloque la thymidylate synthétase, empêchant ainsi la synthèse de novo de thymidine. Cette technique de sélection va stimuler de nombreuses études de génétique somatique, d'autant plus qu'Ephrussi et Mary Weiss montrent que des cellules hybrides somatiques inter-espèces peuvent être obtenues, ouvrant la voie aux cartographies génétiques [56].

II restait un second défi à relever : celui de la très faible fréquence de fusions spontanées entre cellules de différentes lignées lorsqu'elles sont mises en co-culture. Le bond en avant viendra du Japon. En 1958 et les années 




Figure 3. Principe de sélection des hybridomes en milieu HAT. Les cellules de myélome HGPRT- utilisées, adaptées à la culture in vitro, ont été sélectionnées à l'aide de la 8-azaguanine (8-aza) de façon à obtenir des mutants résistants à l'aminoptérine car n'utilisant plus la voie alterne de synthèse des nucléotides du fait de l'inactivation de l'enzyme HPGRT (ou HPRT) (hypoxanthine-guanine phosphoribosyl transférase). La 8-aza, un analogue des purines qui interfère avec la voie alterne, tue en effet les cellules de type sauvage mais les cellules HGPRT- qui apparaissent à la suite de mutations spontanées résistent à cette drogue. Les cellules de myélome HGPRT' sont fusionnées à l'aide de PEG avec des lymphocytes B provenant de souris (ou de rats ou de lapins) ou d'individus immunisés ayant développé une réponse anticorps contre l'antigène d'intérêt. Les cellules hybrides (hybridomes) sont sélectionnées en ajoutant de l'aminoptérine dans le milieu de culture. Les lymphocytes B n'ayant pas fusionné sont incapables de survivre et de proliférer in vitro. Les cellules de myélome non fusionnées sont tuées par l'aminoptérine. Les seules cellules qui survivent sont les cellules hybrides qui possèdent à nouveau un gène sauvage codant I'HGPRT provenant du génome du lymphocyte B fusionné.

suivantes, Yoshio Okada, à l'Institut de recherche sur les maladies microbiennes (RIMD) d'Osaka, montre l'existence d'un virus, le virus de Sendaï, capable de fusionner les membranes plasmiques de cellules tumorales de mammifères [57]. L'utilisation du virus de Sendaï, inactivé par exposition aux rayons ultra-violets (UV), a alors permis d'obtenir plus facilement des cellules hybrides et a été rapidement adopté pour les études de génétique somatique des cellules de mammifères.

Ainsi donc, toutes les pièces du puzzle étaient désormais disponibles pour la découverte de la technique des anticorps monoclonaux par Köhler et Milstein (Figure l et Figure 4)!

\section{Acte V, l'acte final : l'obtention d'anticorps monoclonaux de spécificité prédéfinie !}

César Milstein, dans sa conférence donnée lors de la remise du prix Nobel [58], expliqua qu'il avait rencontré Köhler lors d'une visite à l'Institut d'immunologie de Bâle en Suisse et l'avait fait venir à Cambridge pour étudier les mutations somatiques affectant les anticorps dans les lym- phocytes B. Cependant, cette recherche de mutants ne donnant pas de résultats probants, ils se mirent à chercher un modèle expérimental où l'utilisation de cellules de myélome leur permettrait d'isoler des mutants somatiques dans les régions variables, comme l'avait montré Scharff quelques années plus tôt $[47,48]$. Ils décidèrent alors d'utiliser les cellules du myélome MOPC 21 à cette fin mais aucune spécificité antigénique ne lui étant associée, ils ne réussirent toujours pas à isoler de mutants [58]. Mais c'est de cet échec que leur vint une nouvelle idée qui allait s'avérer être révolutionnaire : outre ses études sur les mutations somatiques, il était prévu que Köhler continue aussi le travail initié par Milstein avec Cotton sur l'étude de l'expression des chaînes d'Ig dans des cellules hybrides provenant de la fusion de cellules de myélome et dont certains d'entre eux produisaient des Ig mutées. Puisqu'ils voulaient obtenir des lignées produisant un anticorps ayant une spécificité connue afin 


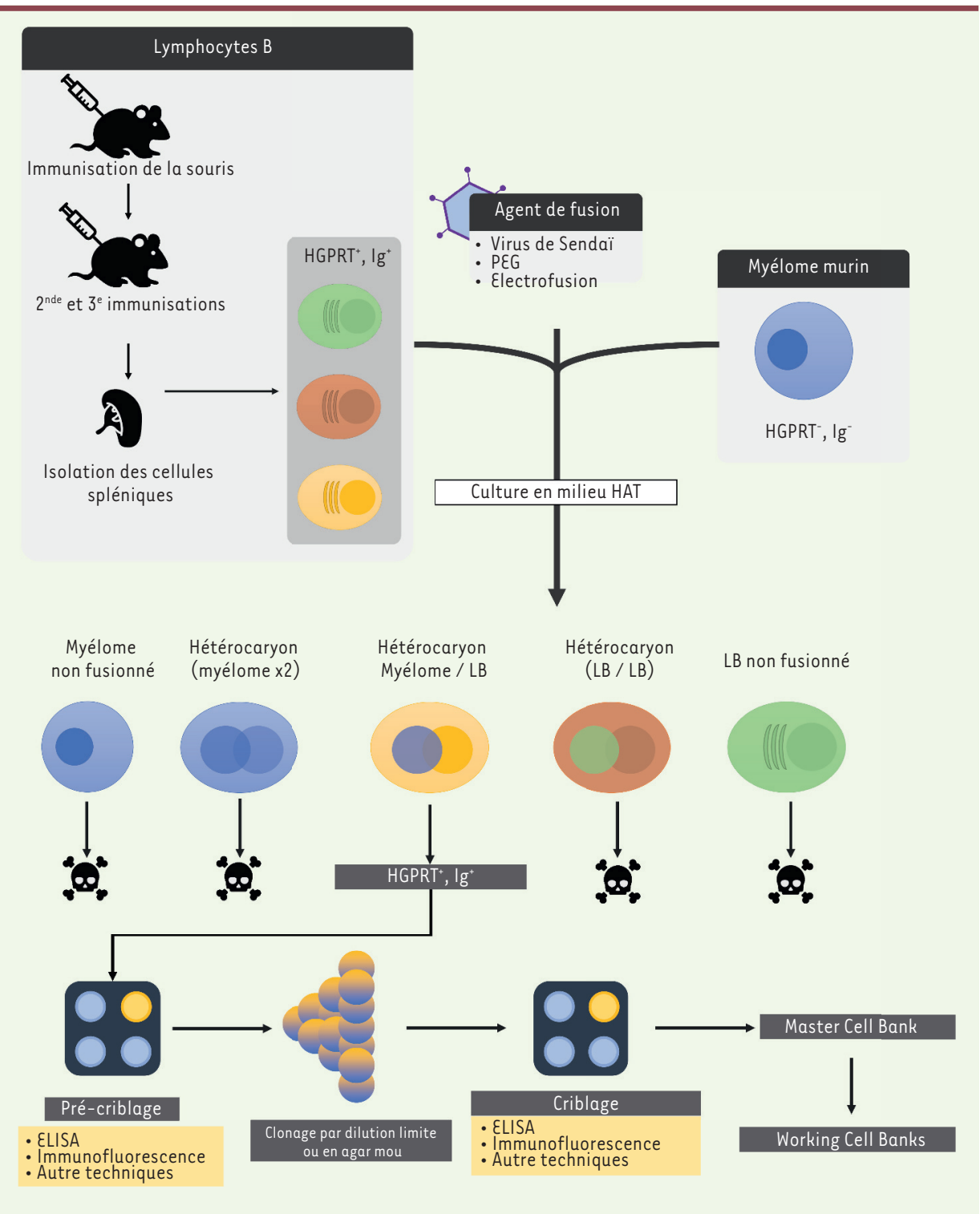

Figure 4. Méthode de production des anticorps monoclonaux. Les lymphocytes spléniques (contenant les plasmablastes et les plasmocytes produisant l'Ac d'intérêt), provenant d'animaux hyperimmunisés sont fusionnés avec des cellules de myélome (HGPRT-, Ig-) avec du polyéthylène glycol (PEG) (initialement avec le virus de Sendaï [1]). La sélection s'effectue en ajoutant de l'aminoptérine dans le milieu de culture, qui contient aussi de l'hypoxanthine et de la thymidine (milieu HAT). Les surnageants de culture des hybridomes (qui sont des « hétérocaryons ») obtenus après environ 2 semaines de culture sont testés le plus souvent d'abord par ELISA (enzyme-linked immunosorbent assay) mais d'autres techniques (immunofluorescence, western-blot, essais fonctionnels, etc.) peuvent être employées en fonction des caractéristiques de I'AcM souhaitées. Les hybridomes correspondants sont alors ensuite clonés, le plus souvent par dilution limite (un clonage en agar mou est aussi utilisé dans quelques laboratoires). Les clones producteurs de I'AcM contre l'antigène d'intérêt sont ensuite cultivés en masse pour créer une master cell bank (MCB) de plusieurs dizaines de cryotubes qui seront répartis sur trois continents lorsqu'il s'agira d'une production industrielle. Des working cell

banks (WCB) sont alors dérivées des cryotubes de la MCB pour la production de l'AcM à une échelle industrielle. Les cellules utilisées pour effectuer les fusions pour obtenir des hybridomes sont le plus souvent les cellules de la lignée X63-Ag8.653 (dérivées de P3/X63-Ag8). Cette lignée de myélome a été dérivée en plusieurs étapes à partir de la lignée du myélome MOPC 21. Les cellules X63-Ag8.653 sont HGPRT' et ne produisent plus l'IgG1, $\kappa$ de MOPC 21

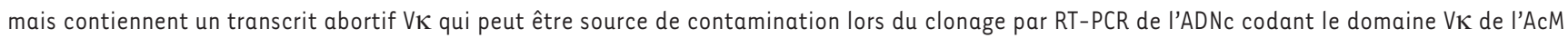
produit par l'hybridome. Il existe d'autres partenaires de fusion comme P3/NS-1.1.Ag4-1 (appelé communément NS-1) (dérivé de P3.X63-Ag8, produisant une chaîne légère $\kappa$ ) ou SP2/0-Agl4 (appelé communément SP2/0), un hybridome (contrairement à ce qui s'écrit très souvent où il est qualifié de myélome), issus de la fusion d'un lymphocyte B avec X63-Ag8.653 et ayant perdu l'expression de ses chaînes lourdes et légères et sélectionné pour sa résistance à la 8-aza et donc HGPRT'.

d'étudier les mutations somatiques affectant cette reconnaissance, Köhler et Milstein décidèrent de fusionner à l'aide du virus de Sendaï des cellules dérivées du myélome MOPC 21, P3-X63Ag8 (dérivées de la lignée P3 elle-même dérivée de MOPC 21) [59], résistante à la 8 -azaguanine et incapable de survivre in vitro en milieu HAT, avec des lymphocytes $B$ de la rate d'une souris BALB/c immunisée contre des globules rouges de mouton (GRM) [1]. La technique utilisée était plus simple que celle utilisée auparavant pour fusionner des cellules de myélomes entre eux ou avec des fibroblastes car il suffisait de tuer les cellules du myélome et de n'utiliser qu'un seul agent de sélection, l'aminoptérine, les lymphocytes B provenant de la souris immunisée et non fusionnés étant incapables de survivre in vitro (Figure 3 et Figure 4). 


\section{Anticorps monoclonaux et brevets : to be or... not to be !}

La découverte révolutionnaire de Köhler et Milstein ne fît pas l'objet d'un brevet. Contrairement à ce qui a été parfois faussement écrit, ils ne s'étaient pas désintéressés de la question. Milstein, à la demande de Tony Vickers, un officiel du Medical Research Council (MRC) lui avait transmis le manuscrit qu'il destinait à la revue Nature avant sa publication. Celui-ci fut alors transmis au National Research and Development Corporation (NRDC), l'agence gouvernementale britannique, séparée du MRC, et qui était seule responsable des dépôts de brevets, pour évaluer si la découverte décrite dans le manuscrit pouvait faire l'objet d'une demande de brevet. La réponse du NRDC (Figure 5) que rapporte Milstein [59], fut que « le travail tel que publié n'était pas brevetable. Un travail supplémentaire considérable devrait être effectué dans des conditions de secret pour développer l'invention jusqu'à un stade où elle pourrait être protégée ».* Avec humour, Milstein fait le commentaire suivant [59]: «Ainsi, avec du recul, nous pouvons nous considérer comme extrêmement chanceux que, pour une raison qui m'échappe, on ne nous ait jamais demandé de travailler en secret ou de ne pas envoyer la lignée de myélome X63 que nous avions utilisée comme partenaire de fusion à nos collègues du monde entier ».** Effectivement, Milstein envoya la lignée de myélome X63Ag8 à tous les chercheurs académiques qui le lui demandèrent, notamment à Hilary Koprowski au Wistar Institute de Philadelphie qui... obtint, avec Carlo Croce,

le premier brevet sur la fabrication d'anticorps monoclonaux (en l'occurrence contre les antigènes tumoraux) le 23 octobre 1979 , malgré la demande de Milstein lors de son envoi de la lignée que «les produits obtenus en utilisant ces cellules ne devront pas faire l'objet de brevets ». Ce dernier ne retrouva malheureusement pas la trace de cette demande, si ce n'est indirectement dans une lettre adressée à Koprowski en réponse à une autre demande de ce dernier de transférer la lignée X63 à l'un de ses collègue, lettre envoyée un peu avant le dépôt de la demande du Wistar Institute (Figure 6) ! Heureusement pour la communauté scientifique mondiale, l'absence d'un brevet princeps et la générosité de Milstein permirent la diffusion rapide de la technique et d'engranger des progrès considérables dans de nombreuses disciplines de la biologie et bientôt de la médecine.

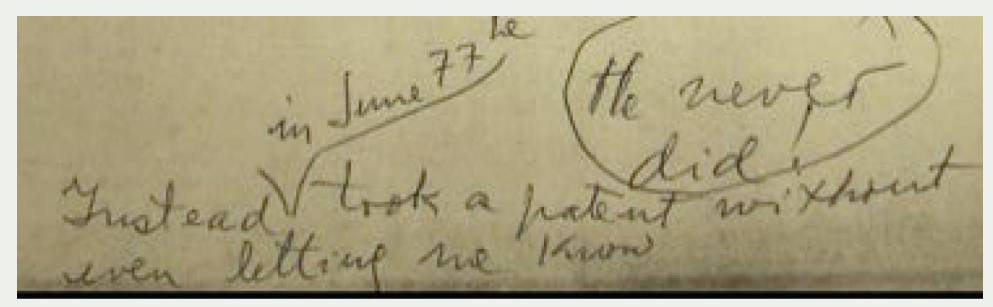

Figure 6. Commentaires de Milstein sur la lettre que lui écrivit Koprowski en novembre 1976, quelques temps après qu'il ait appris qu'une demande de brevet avait été déposée par l'organisme de tutelle de ce dernier, le Wistar Institute (source : Churchill Archives Centre, Churchill College, Milstein's Papers, file MSTN/C324).

* "They expressed the view that the "work as published was not patentable. Considerable further work would have to be carried out in conditions of secrecy to develop the invention to a state when it could have been adequately protected » [59].

${ }^{* \star}$ «So, with hindsight, we may have been extremely lucky that, for one reason or another, we were never asked to work in secrecy or to refrain from sending the X63 myeloma partner to our colleagues all over the world $\gg[59]$. 
Après le clonage des cellules hybrides en agar mou, les clones (appelés $\mathrm{Sp}-1,-2$, etc.) furent testés pour leur production d'anticorps anti-GRM par un test de plages de lyse. Une fois établie la stabilité des clones en culture pendant 4 mois, l'analyse de leur caryotype montra qu'ils contenaient un nombre de chromosomes très supérieur à celui d'une seule cellule splénique de souris BALB/c mais inférieur à la somme des chromosomes de deux cellules de myélome, ainsi qu'un chromosome métacentrique présent dans MOPC 21 , ce qui était en faveur du fait que les cellules hybrides obtenues n'étaient pas issues de la fusion de deux lymphocytes $B$ ou de deux cellules de myélome, mais bien d'une cellule du myélome P3-X63Ag8 et d'un lymphocyte B, héritant de la capacité des cellules du myélome à se multiplier indéfiniment et du lymphocyte $B$ fusionné à fabriquer un anticorps anti-GRM. Ces cellules donnant naissance à des populations de cellules filles identiques entre elles, chaque clone de cellules filles produisait le même anticorps, donc monoclonal. Les hybridomes ${ }^{9}$ et les anticorps monoclonaux étaient nés! (Figure 4). L'utilisation d'un myélome producteur d'une immunoglobuline fut l'objet d'un questionnement quant aux immunoglobulines produites puisque les cellules du myélome parental utilisé pour la fusion, X63Ag8 ${ }^{10}$, produisaient I'IgGl $\kappa$ de MOPC21. Il y avait donc dans ces hybrides les chaînes $H$ et $L$ de MOPC 21 ainsi que celles des lymphocytes $B$ fusionnés, comme le montrèrent Köhler et Milstein par des analyses de focalisation isoélectrique (isoelectrofocusing [IEF]) [1], et donc des formes H2L2 hétérologues résultant de l'association aléatoire de ces chaînes! Seules une partie d'entre elles étaient des anticorps anti-GRM provenant des lymphocytes $B$ de la souris immunisée, le reste incluant l'Ig MOPC 21 et des formes « hybrides » où les chaînes H provenant de MOPC 21 étaient associées à des chaînes L provenant du lymphocyte $B$, et réciproquement. Ce problème fut résolu dans les années suivantes par l'utilisation de variants, notamment Sp2/0-Agl4, un clone d'hybridome anti-GRM provenant de la fusion d'un lymphocyte $B$ avec X63-Ag8, ayant perdu la capacité de synthétiser ses chaînes $\mathrm{H}$ et $L$ [61]. Les mois qui suivirent la publication de Köhler et Milstein virent par ailleurs une amélioration importante de la technique de fusion cellulaire grâce à un chimiste italien chassé de l'université de Florence en 1938 du fait de ses origines juives, qui était parti en 1939 au RoyaumeUni, Guido Pontecorvo. Celui-ci montra que le polyéthylène glycol (PEG) permettait de fusionner les membranes cellulaires sans effet cytopathique (Figure 4) [62]. Cette découverte eut de nombreuses applications non seulement pour créer des cellules hybrides, mais aussi pour mettre au point des techniques de transfection de matériel génétique dans les cellules eucaryotes.

\section{Conclusion}

La mise au point de la technique des anticorps monoclonaux est issue de la très longue histoire de la recherche sur les lg et les anticorps

\footnotetext{
${ }^{9}$ Ce nom, proposé par Len Herzenberg (université de Stanford) pendant son séjour sabbatique dans le laboratoire de Milstein en 1976-1977 [59], apparaîtra pour la première fois dans une publication de Köhler en février 1978 [60], les premières cellules issues des fusions étant désignées auparavant comme étant des « cellules hybrides » ou « hybrides » ou « hybrides avec des lignées cellulaires de myélome ».

${ }^{10}$ Désignée également parfois sous le nom de P3-X67Ag8 dans l'article de Köhler et Milstein [1] !
}

commencée à la fin du XIX siècle. Elle est le produit de recherches très fondamentales, académiques, qui ont mobilisé des chercheurs du monde entier, des « fous de science » qui ont construit pendant des décennies tout au long d'une grande partie du $x^{e}$ siècle, morceau après morceau, le puzzle que l'intelligence de Georges Köhler et César Milstein a su finalement reconstituer pour donner au monde l'accès à une nouvelle classe de médicaments et à des outils diagnostiques qui ont révolutionné et continuent de révolutionner la médecine et le traitement de pathologies toujours plus nombreuses. $\diamond$

\section{SUMMARY}

MAbs: the history of a basic research or the curiosity as a source of wealth

In 2019, monoclonal antibodies are a worldwide annual business worth of more than 100 billions USD (i.e., about 90 billions $€$ ). In addition to their use in the clinics, monoclonal antibodies are also used for diagnosis and remain highly valuable tools for academic basic and translational research. Forty-four years after the seminal publication of Georges Köhler and César Milstein, dozens of meetings and seminars focusing on various aspects of mAbs are held annually all around the world. But forty-four years later, the scientific works and efforts that have made possible this scientific breakthrough are gradually forgotten and, for many, monoclonal antibodies are no more than a multi-million USD business alike any other big business, guided by financial markets and the results of on-going clinical trials... Time has now come for acknowledging and paying tribute to all these scientists involved in basic research, to these researchers passionate about science, some famous, some forgotten, scattered all over the world. They explored during the $20^{\text {th }}$ century the frontiers of unknown and generated a knowledge that allowed the emergence of a technique that translated finally into what is one of the greatest therapeutic revolution of the modern era. $\diamond$

\section{LIENS D'INTÉRÊT}

Les auteurs déclarent n'avoir aucun lien d'intérêt concernant les données publiées dans cet article.

\section{RÉFÉRENCES}

1. Köhler G, Milstein C. Continuous cultures of fused cells secreting antibody of predefined specificity. Nature $1975 ; 256: 495-7$.

2. Ehrlich P, Kossel H, Wassermann A. Collected papers, vol. II, pp. 56-60. Deutsch Med Wochenschr $1894 ; 20: 353$.

3. Landsteiner $\mathrm{K}$, van der Scheer J. On the specificity of agglutinins and precipitins. J Exp Med $1924 ; 40: 91-107$.

4. Landsteiner K, Simms S. Production of heterogenetic antibodies with mixtures of the binding part of the antigen and protein. J Exp Med 1923; $38: 127-38$.

5. Grabar P, Williams CW. Method permitting the combined study of the electrophoretic and the immunochemical properties of protein mixtures ; application to blood serum. Biochim, Biophys Acta 1953 ; 10 : 193-4. 


\section{RÉFÉRENCES}

6. Heidelberger M, Pedersen KO. The molecular weight of antibodies. J Exp Med 1937 ; 65 : 393-414.

7. Tiselius $A$, Kabat $\varepsilon A$. An electrophoretic study of immune sera and purified antibody preparations. J Exp Med 1939; 69 : 119-31.

8. McFarlane Burnet FM. A modification of Jerne's theory of antibody production using the concept of clonal selection. Austral J Sci $1957 ; 20: 67-9$.

9. Nossal GJV, Lederberg J. Antibody production by single cells. Nature $1958 ; 181: 1419-20$.

10. Pauling L. A theory of the structure and process of formation of antibodies. J Am Chem Soc 1940 ; $62: 2643-57$

11. Porter RR. Separation and isolation of fractions of rabbit gamma-globulin containing the antibody and antigenic combining sites. Nature $1958 ; 182: 670-1$.

12. Porter RR. The hydrolysis of rabbit $\gamma$-globulin and antibodies with crystalline papain. Biochem J $1959 ; 73: 119-26$.

13. Edelman GM, Poulik MD. Studies on structural units of the $\gamma$-globulins. J Exp Med 1961;61-84

14. Porter RR. Symposium on basic problems in neoplastic disease. In : Gellhorn A, Hirschberg $\varepsilon$. New York : Columbia University Press, $1962: 177$.

15. Pain RH. The molecular weights of the peptide chains of $\gamma$-globulins. Biochem J $1963 ; 88: 234-9$.

16. Edelman GM, Benacerraf B, Ovary Z, Poulik MD. Structural differences among antibodies of different specificities. Proc Natl Acad Sci USA $1961 ; 47: 1751-8$.

17. Fleischman JB, Porter RR, Press EM. The Arrangement of the peptide chains in $\gamma$-Globulin. Biochem J $1963 ; 88: 220-8$.

18. Edelman GM, Olins DE, Gally JA, Zinder ND. Reconstitution of immunologic activity by interaction of polypeptide chains of antibodies. Proc Natl Acad Sci USA 1963 ; 50 : 753-61.

19. Fougereau $M$, Olins $D E$, Edelman $G M$. Reconstitution of antiphage antibodies from $L$ and $H$ polypeptide chains and the formation of interspecies molecular hybrids. J Exp Med $1964 ; 120$ : 349 58.

20. Edelman GM, Gally JA. The nature of Bence-Jones proteins - chemical similarities to polypeptide chains of myeloma globulins and normal $\gamma$-globulins. J Exp Med $1962 ; 116: 207-27$.

21. Potter M, Fahey JL, Pilgrim HI. Abnormal serum protein and bone destruction in transmissible mouse plasma cell neoplasm (multiple myeloma). Proc Soc Exp Biol Med 1957 ; 94 : 327-33.

22. Nathans $D$, Fahey JL, Potter M. The formation of myeloma protein by a mouse plasma cell tumor. J Exp Med $1958 ; 108: 121-30$.

23. Askonas B, Fahey JL. Formation of Bence-Jones protein and myeloma protein in vitro by the plasmacell tumor MPC-2. Biochem/ $1961 ; 80: 261-8$.

24. Merwin RM, Algire $H$. Induction of plasma-cell neoplasms and fibrosarcomas in BALB/c mice carrying diffusion chambers. Proc Soc Exp Biol Med $1959 ; 101$ : 437-9.

25. Potter M, Robertson CL. Development of plasma-cell neoplasms in BALB/c mice after intraperitoneal injection of paraffin-oil adjuvant, heart-killed Staphylococcus mixtures. J Natl Cancer Inst 1960 ; $25: 847-61$.

26. Potter $M$, Boyce CR. Induction of plasma-cell neoplasms in strain BALB/c mice with mineral oil and mineral oil adjuvants. Nature $1962 ; 193: 1086-7$

27. Evans VJ, LaRock JF, Yosida TH, Potter M. A new tissue culture isolation and explantation of the P388 lymphocytic neoplasm in a chemically characterized medium. Exp Cell Res $1963 ; 32: 212-7$.

28. Laskov R, Scharff MD. Synthesis, assembly, and secretion of gamma globulin by mouse myeloma cells. I. Adaptation of the Merwin plasma cell tumor-11 to culture, cloning, and characterization of gamma globulin subunits. J Exp Med 1970 ; 131 : 515-41.

29. Horibata K, Harris AW. Mouse myelomas and lymphomas in culture. Exp Cell Res $1970 ; 60: 61-77$

30. Bennett JC, Hood LE, Dreyer WJ, Potter W. Evidence for amino-acid sequence differences among proteins resembling the L-chain subunits of immunoglobulins. J Mol Biol $1965 ; 12$ : 81-7.

31. Hilschmann N, Craig LC. Amino acid sequence studies with Bence Jones proteins. Proc Natl Acad Sci USA $1965 ; 53: 1403-9$

32. Koshland $M E$, Englberger FM. Differences in the amino acid composition of two purified antibodies from the same rabbit. Proc Natl Acad Sci USA 1963; $50: 61-8$.

33. Singer SJ, Doolittle RF. Antibody active sites and immunoglobulin molecules. Science 1966 ; 153 : $13-25$.

34. Edelman GM, Gally JA. Somatic recombination of duplicated genes: an hypothesis on the origin of antibody diversity. Proc Natl Acad Sci USA $1967 ; 57: 353-8$.

35. Brenner S, Milstein C. Origin of antibody variation. Nature $1966 ; 211: 242-3$.

36. Gottlieb PD, Cunningham BA, Waxdal MJ, Konigsberg WH, Edelman GM. Variable regions of heavy and light polypeptide chains of the same $\gamma \mathrm{G}$-immunoglobulin molecule. Proc Natl Acad Sci USA 1968 ; $61: 168-75$.

37. Singer SJ, Thorpe NO. On the location and structure of the active sites of antibody molecules. Proc Natl Acad Sci USA $1968 ; 60: 1371-8$
38. Milstein C. Linked groups of residues in immunoglobulin $\mathrm{K}$ chains. Nature $1967 ; 216: 330-2$

39. Cunningham BA, Pflumm MN, Rutishauser U, Edelman GM. Subgroups of amino acid sequences in the variable regions of immunoglobulin heavy chains. Proc Natl Acad Sci USA 1969 ; 64 : 997-1003.

40. Dreyer WJ, Bennett CJ. The molecular basis of antibody formation: a paradox. Proc Natl Acad Sci USA $1965 ; 54: 864-9$.

41. Wu T, Kabat $\varepsilon A$. An analysis of the sequences of the variable regions of Bence Jones proteins and myeloma light chains and their implications for antibody complementarity. J Exp Med 1970 ; 132 : 211-50.

42. Oudin J. Allotypy of rabbit serum proteins. II. Relationships between various allotypes: their common antigenic specificity, their distribution in a sample population. Genetic implications. J Exp Med 1960 ; 112 : 125-42.

43. Oudin J. Genetic regulation of immunoglobulin synthesis. J Cell Physiol 1966 ; 67 (suppl 1) : 77-108.

44. Oudin J, Michel M. De la spécificité idiotypique des anticorps de lapin anti-S typhi. CR Hebd Acad Sci Seances Acad Sci Serie D. $1969 ; 268: 230-3$.

45. Edelman GM, Cunningham BA, Gall WE, Gottlieb PD, Rutishauser U, Waxdal MJ. The covalent structure of an entire $\mathrm{gG}$ immunoglobulin molecule. Proc Natl Acad Sci USA $1969 ; 63: 78-85$.

46. de Préval C, Pink JR, Milstein C. Variability of interchain binding of immunoglobulins. Interchain bridges of mouse IgG2a and IgG2b. Nature 1970 ; $228: 930-2$.

47. Coffino P, Scharff MD. Rate of somatic mutation in immunoglobulin production by mouse myeloma cells. Proc Natl Acad Sci USA 1971 ; $68: 219-23$.

48. Coffino P, Laskov R, Scharff MD. Immunoglobulin production : method for quantitively detecting variant myeloma cells. Science $1970 ; 167: 186-8$.

49. Secher DS, Cotton RGH, Milstein C. Spontaneous mutation in tissue culture chemical nature of variant immunoglobulin from mutant clones of MOPC 21. FEBS Letter 1973 ; 37 : 311-6.

50. Periman P. IgG synthesis in hybrid cells from an antibody-producing mouse myeloma and an L cell substrain. Nature 1970 ; 228 : 1086-7.

51. Coffino P, Knowles B, Nathenson SG, Scharff MD. Suppression of immunoglobulin synthesis by cellular hybridization. Nat New Biol $1971 ; 231$ : 87-90.

52. Finch BW, Ephrussi B. Retention of multiple developmental potentialities by cells of a mouse testicular teratocarcinoma during prolonged culture in vitro and their extinction upon hybridization with cells of permanent lines. Proc Natl Acad Sci USA 1967; $57: 615-21$.

53. Cotton RG, Milstein C. Letter: Fusion of two immunoglobulin-producing myeloma cells. Nature $1973 ; 244: 42-3$

54. Barski G, Sorieul S, Cornefert F. Production of cells of a hybrid nature in culture in vitro of 2 cellular strains in combination. CR Hebd Seances Acad Sci 1960 ; $251: 1825-7$.

55. Littlefield JW. Selection of hybrids from matings of fibroblasts in vitro and their presumed recombinants. Science 1964 ; 145 : 709-10.

56. Ephrussi B, Weiss MC. Interspecific hybridization of somatic cells. Proc Natl Acad Sci USA $1965 ; 53: 1040-2$.

57. Okada Y. The fusion of Ehrlich's tumor cells caused by HVJ virus in vitro. Biken J $1958 ; 1: 103-10$

58. Milstein C. From the structure of antibodies to the diversification of the immune response. Nobel lecture 8 december 1984. Biosci Rep 1984 ; 4 : 275-97 et Embo J $1985 ; 4: 1083-92$.

59. Milstein C. The hybridoma revolution: an offshoot of basic research. BioEssays $1999 ; 21: 966-73$.

60. Köhler G, Hengartner H, Shulman MJ. Immunoglobulin production by lymphocyte hybridomas. Eur J Immunol $1978 ; 8: 82-8$.

61. Shulman M, Wilde CD, Köhler G. A better cell line for making hybridomas secreting specific antibodies. Nature $1978 ; 276: 269-70$.

62. Pontecorvo $G$. Production of mammalian somatic cell hybrids by means of polyethylene glycol treatment. Somat Cell Genet $1975 ; 1: 397-400$.

\section{TIRÉS À PART}

J.L. Teillaud

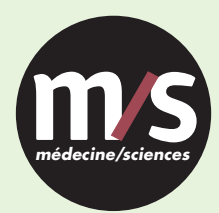

Tarifs d'abonnement $\mathrm{m} / \mathrm{s}-2019$

Abonnez-vous

à médecine/sciences
$>$ Grâce à $\mathrm{m} / \mathrm{s}$, vivez en direct les progrès des sciences biologiques et médicales

Bulletin d'abonnement page 1230 dans ce numéro de $\mathrm{m} / \mathrm{s}$

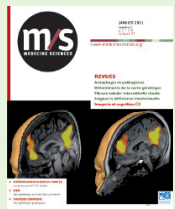

